\title{
A NOTE ON RIGHT EQUIVALENCE OF MODULE PRESENTATIONS
}

\author{
M. J. CANFELL
}

(Received 28 July 1989; revised 27 November 1990)

Communicated by B. J. Gardner

\begin{abstract}
If $R$ has 1 in the stable range, then any two presentations $f, g: P \rightarrow M$ of an $R$-module $M$ by a finitely generated projective $P$ are right equivalent, that is, $f=g h$ for some automorphism $h$ of $P$. The converse is also true.

1991 Mathematics subject classification (Amer. Math. Soc.) 16 A 48.
\end{abstract}

Let $R$ be an associative ring with 1 . All modules considered will be unital right $R$-modules, and all homomorphisms will act on the left.

Two module epimorphisms $f, g: P \rightarrow M$ are right equivalent if $f=g h$ for some automorphism $h$ of $P$.

After Bass, we say that $R$ has 1 in the stable range if $a, b \in R$ and $a R+b R=R$ imply $a+b t$ is a unit of $R$ for some $t \in R$.

For $P=R^{n}$, the implication (a) $\Rightarrow$ (b) below is due to Warfield [2], [3]. The methods used there are different. In fact the proof in [2] is incorrect, while the proof in [3] involves the substitution property. We give a simple direct proof and also show that the converse implication holds.

THEOREM. The following conditions are equivalent.

(a) $R$ has 1 in the stable range.

(b) Each pair of epimorphisms $f, g: P \rightarrow M$ from a finitely generated projective $R$-module $P$ to an $R$-module $M$ are right equivalent.

(C) 1992 Australian Mathematical Society $0263-6115 / 92 \$ A 2.00+0.00$ 
Proof. (a) $\Rightarrow$ (b) Assume (a) and let $P$ be a finitely generated projective $R$-module. By [1, Corollary 2.9], the endomorphism ring $\operatorname{End}(P)$ also has 1 in the stable range. Given epimorphisms $f, g: P \rightarrow M$, choose $h_{1}, h_{2} \in$ $\operatorname{End}(P)$ such that $g h_{1}=f$ and $f h_{2}=g$. Thus $g\left(1-h_{1} h_{2}\right)=0$. Since $h_{1} h_{2}+\left(1-h_{1} h_{2}\right)=1$ and $\operatorname{End}(P)$ has 1 in the stable range, there is a $k \in \operatorname{End}(P)$ such that $h=h_{1}+\left(1-h_{1} h_{2}\right) k$ is an automorphism of $P$. Also $g h=f$ and so $f, g$ are right equivalent.

(b) $\Rightarrow$ (a) Assume (b) holds and suppose $a, b \in R$ satisfy $a R+b R=R$. Choose $s, t \in R$ such that $a s+b t=1$. Let $M=R / b R$ and let $g: R \rightarrow M$ be the canonical map. Define $f: R \rightarrow M$ by $f(r)=g(a r)$. Since $g(1)=$ $g(a s+b t)=g(a s)=f(s)$ we see that $f$ is an epimorphism. By assumption there is an automorphism $h$ of $R$ such that $g h=f$. Now $h(1)$ is a unit of $R$ and $g h(1)=f(1)=g(a)$. Thus $h(1)-a \in \operatorname{Ker} g=b R$ so $a+b t$ is a unit for some $t \in R$.

\section{References}

[1] L. N. Vaserstein, 'Bass's first stable range condition', J. Pure Appl. Algebra 34 (1984), 319-330.

[2] R. B. Warfield, Jr., 'Stable equivalence of matrices and resolutions', Comm. Algebra 6 (1978), 1811-1828.

[3] R. B. Warfield, Jr., 'Stable generation of modules', Lecture Notes in Mathematics 700, pp. 16-33 (Springer-Verlag, New York, 1979).

Department of Mathematics, Statistics and Computing Science University of New England

Armidale, N.S.W. 2351

Australia 